\title{
MODELING, ANALYSIS AND COMPUTATION OF FLUID STRUCTURE INTERACTION MODELS FOR BIOLOGICAL SYSTEMS
}

\author{
S. Minerva Venuti \\ Department of Mathematical Sciences \\ George Mason University, Fairfax VA 22030 \\ email: swelling@gmu.edu \\ Sponsor: Dr. Padmanabhan Seshaiyer \\ Department of Mathematical Sciences \\ George Mason University, Fairfax VA 22030 \\ email: pseshaiy@gmu.edu
}

\begin{abstract}
A mathematical modeling for the interaction of blood flow with the arterial wall surrounded by cerebral spinal fluid is developed. The blood pressure acting on the inner arterial wall is modeled using a Fourier Series, the arterial wall is modeled using a spring-mass system, and the surrounding cerebral spinal fluid is modeled via a simplified Navier-Stokes equation. The resulting coupled system of partial differential equations for this fluid structure interaction with appropriate boundary conditions are solved first analytically using Laplace Transform and then numerically using an implicit finite difference scheme. The solutions are also investigated using computational tools. An application of the model studied to intracranial saccular aneurysms is also presented.
\end{abstract}

\section{INTRODUCTION}

Fluid structure interaction models can be used to gain insight into a number of different applications, such as the interaction between airflow and wings of microair vehicles and blood pressure interaction with arterial walls $[5,1,18]$. This paper focuses on modeling an intracranial saccular aneurysm, which is a focal dilatation of an arterial wall within the brain. Between 2 and $5 \%$ of the population harbor aneurysms within their brains and 15 to $30 \%$ of those that harbor at least one aneurysm have multiple lesions $[3,7,10]$.

While there have been a number of papers written about intracranial saccular aneurysm, specific mechanisms responsible for their genesis, enlargement, and rupture remain unknown $[12,8,14,15]$. It has been hypothesized that one of the reasons for a saccular aneurysm to enlarge and rupture is because the dynamic behavior of the arterial wall is unstable because of the pulsatile blood flow $[9,17,13]$. To investigate this hypothesis, we will build a one dimensional coupled model of an intracranial saccular aneurysm herein, that incorporates the interaction between the blood pressure, the wall structure, and the cerebral spinal fluid that surrounds 


\section{S. MINERVA VENUTI}

the aneurysm. While this one dimensional model may be a simplification of a complex biological problem, it does give us insight to what is happening with the interactions.

Toward this end, we derive a coupled system of equations of motion for an idealized subclass of lesions. The blood pressure acting on the inner arterial wall is modeled using a Fourier Series. The arterial wall is modeled using a spring-mass system. The surrounding cerebral spinal fluid is modeled via a simplified Navier-Stokes equation. We then use both analytical and numerical methods to derive exact solutions that will examine the response of this subclass of lesions against imposed pulsatile blood flow.

\section{Mathematical Models and Background}

The problem we consider models three components of the intracranial saccular aneurysm - the blood pressure acting on the inside of the arterial wall, the structure of the arterial wall, and the cerebral spinal fluid (CSF) that surrounds the aneurysm (see figure 1a). To derive the one dimensional model, we consider a line that runs from within the aneurysm through the arterial wall and out into the CSF, as shown in Figure 1b. We consider the point $x=0$ to be where the outside wall and CSF meet, with $x>0$ to be moving away from the wall into the CSF, and $x<0$ to be moving through the wall and into the aneurysm. Next we outline individual models

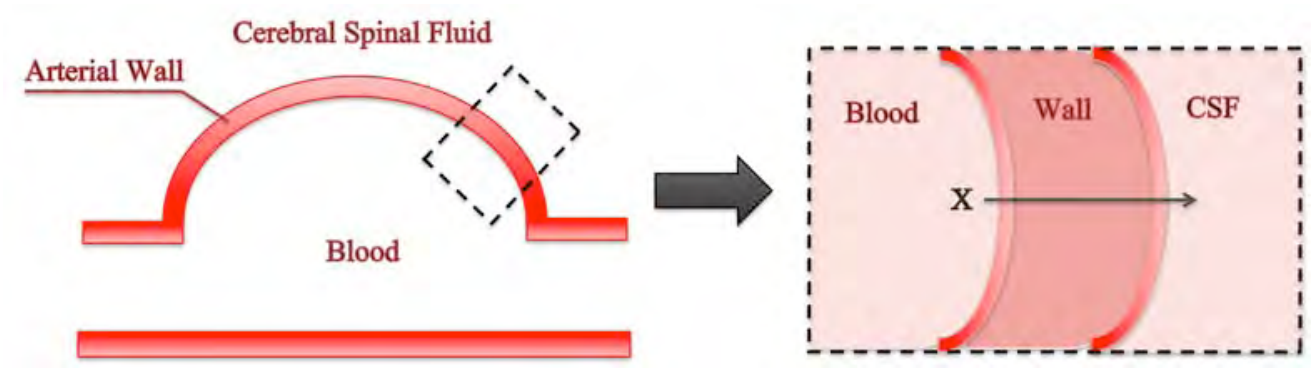

FiguRE 1. Aneurysm with direction $x$ shown

for the CSF, arterial wall, and the blood flow and explain how they will be coupled.

2.1. Model of the cerebral spinal fluid. To model the CSF, we consider the following equation:

$$
\rho v_{t}+\rho v v_{x}+P_{x}-\mu v_{x x}=F .
$$

Note that (1) may been seen as the one dimensional Navier-Stokes equation. Here $\rho$ is density, $v(x, t)$ is the velocity of the fluid, $P(x, t)$ is the pressure of the fluid, $\mu$ is the viscosity of the fluid and $F$ is the body force on the fluid. In order to solve (1) analytically, we need to simplify our model. To do so, we make the following assumptions: that the CSF is slightly compressible and inviscid, that there are no external forces acting on the fluid, and that the nonlinear effects are negligible. The last three (inviscid, no external forces, nonlinear negligible) simplifies eq (1) to:

$$
\rho v_{t}+P_{x}=0
$$


Now notice that there are still two unknowns, the pressure and the velocity. By using our assumption that the CSF is slightly compressible, we can use the state equation for a slightly compressible mixture which is:

$$
\rho_{P}=\rho e^{\gamma^{-1} P(x, t)}
$$

where $\gamma=\rho c^{2}, c$ is the speed of sound through the fluid. Taking the derivative of $\rho_{P}$ with respect to $P$ gives

$$
\frac{d \rho_{P}}{d P}=\left(\frac{\rho}{\gamma}\right) e^{\gamma^{-1} P(x, t)}
$$

Substituting eq (3) into eq (4) gives:

$$
\frac{d \rho_{P}}{d P}=\frac{\rho_{P}}{\gamma} .
$$

The Law of Conservation of Mass gives:

$$
\begin{aligned}
\frac{\partial \rho_{P}}{\partial t} & =-\frac{\partial}{\partial x}\left(\rho_{P} v\right) \\
& =-\rho_{P} \frac{\partial v}{\partial x}-v \frac{\partial \rho_{P}}{\partial x} \\
\frac{d \rho_{P}}{d P} \frac{\partial P}{\partial t} & =-\rho_{P} \frac{\partial v}{\partial x}-v \frac{d \rho_{P}}{d P} \frac{\partial P}{\partial x}
\end{aligned}
$$

by using the product rule and chain rule. Substituting eq (5) into eq (8) we get:

$$
\frac{\rho_{P}}{\gamma} \frac{\partial P}{\partial t}=-\rho_{P} \frac{\partial v}{\partial x}-v \frac{\rho_{P}}{\gamma} \frac{\partial P}{\partial x} .
$$

As $\gamma$ is very large, the second term on the right hand side is very small compared to the first, we can assume it is negligible. By integrating with respect to time, eq (9) reduces to:

$$
\frac{\rho_{P}}{\gamma} \frac{\partial P}{\partial t}=-\rho_{P} \frac{\partial v}{\partial x}
$$

We now introduce a new variable $u(x, t)$ to be the displacement of the fluid. This can be related to the fluid velocity through:

$$
u(x, t)=\int_{0}^{t} v(x, s) d s .
$$

Using this relationship between $u$ and $v$, we can now simplify eq (10) to:

$$
\begin{aligned}
\frac{\rho_{P}}{\gamma} \frac{\partial P}{\partial t} & =-\rho_{P} \frac{\partial u_{t}}{\partial x} \\
\frac{\rho_{P}}{\gamma} \frac{\partial P}{\partial t} & =-\rho_{P} \frac{\partial}{\partial x} \frac{\partial u}{\partial t} \\
\frac{\rho_{P}}{\gamma} P & =-\rho_{P} \frac{\partial u}{\partial x} \\
\frac{P}{\gamma} & =-u_{x} \\
P & =-\rho c^{2} u_{x} .
\end{aligned}
$$

So we now have a relationship between the pressure and displacement. Using this relationship and eq (11) simplifies (2) to:

$$
\begin{aligned}
& v_{t}=c^{2} u_{x x} \\
& u_{t}=v
\end{aligned}
$$




\section{S. MINERVA VENUTI}

Note that together eqs (17) and (18) can be thought of as the standard wave equation. What we are really interested in the movement of the wall, which is the same as the movement of the fluid at the point $x=0$. Thus by finding the solution to eqs (17) and (18) at the point $x=0$ for time $t \geq 0$ we will know the movement of the wall for all time after $t=0$. To solve the partial differential equations, we need two boundary conditions and two initial conditions. For initial conditions, we assume that the CSF starts at rest and has no initial velocity, giving us the equations

$$
u(x, 0)=v(x, 0)=0 .
$$

The two boundary conditions will be developed in the governing equation of motion section, after we develop models for the wall and blood pressure which is presented next.

2.2. Model of the arterial wall. To model the arterial wall, we use a spring and mass system, with the spring constant $k$ and mass $m$. Figure 2 illustrates this coupled system between the inner wall and outer wall. The force generated by this system is given by $k\left(x_{\text {outerwall }}-x_{\text {innerwall }}\right)$ where $x_{\text {outerwall }}$ and $x_{\text {innerwall }}$ are the respective displacements of the inner and outer wall from equilibrium, which will be described later in the governing equation of motion.

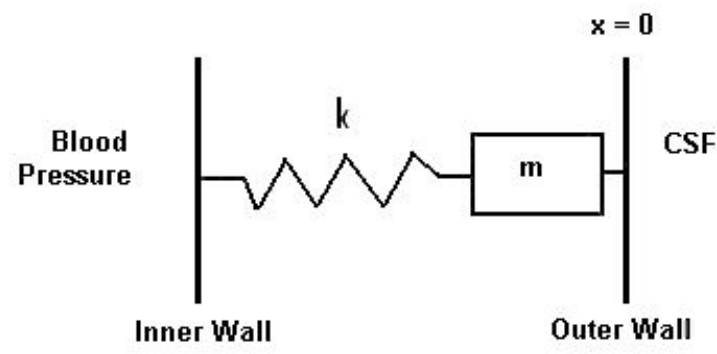

FIGURE 2. Spring and mass system

2.3. Model of the blood pressure. As blood pressure is considered to be pulsatile it can be modeled by the Fourier series $[4,11,16]$ :

$$
P_{\mathrm{BLOOD}}(t)=P_{m}+\sum_{n=1}^{N}\left(A_{n} \cos (n \omega t)+B_{n} \sin (n \omega t)\right)
$$

where $P_{m}$ is the mean blood pressure, and $A_{n}$ and $B_{n}$ are the Fourier coefficients for $N$ harmonics, and $\omega$ is the fundamental circular frequency, which are available in the literature [11].

2.4. Governing Equation of Motion. To solve the coupled system for the partial differential equation (PDE) derived in eqs (17)-(18) we still need two boundary conditions. Our first boundary condition, at the point $x=0$, is derived from our models for the wall and blood pressure. We do this by writing a force balance equation at the point $x=0$, where

$$
F_{\text {TOTAL }}=F_{\text {FLUID }}-F_{\text {SPRING }} \text {. }
$$

The total force $F_{\text {TотаL }}$ is equal to $m v_{t}(0, t)$, where $m$ is the mass of the wall, as shown in Figure 2. The force of the fluid $F_{\text {FLUID }}$ is the pressure of the fluid times the area. 
Using the assumption that the fluid is slightly compressible allows us to rewrite pressure in terms of displacement, giving us $F_{\text {FLUID }}=\rho c^{2} u_{x}(0, t) a$, where $a$ is the cross-sectional area. The force from the spring is $F_{\mathrm{SPRING}}=k\left(x_{\text {outerwall }}-x_{\text {innerwall }}\right)$. Note that the displacement of the outer wall is $u(0, t)$. For the interior wall, as it is affected only by the blood pressure, it's displacement is proportional to the blood pressure. So the total force from the spring becomes

$$
F_{\text {SPRING }}=k u(0, t)-a P_{\text {BLOOD }}(t) .
$$

Thus by combining (20) and (22) we get the following boundary condition from $(21)$

$$
m v_{t}(0, t)=a P_{m}-k u(0, t)+\rho c^{2} a u_{x}(0, t)+\sum_{n=1}^{N}\left(a A_{n} \cos (n \omega t)+a B_{n} \sin (n \omega t)\right) .
$$

For the second boundary condition, we use the plane wave approximation, which says that the waves from the wall will die down some long distance away from the wall, which we call the point $x=L$. This is given by

$$
v(L, t)=-c u_{x}(L, t) \text {. }
$$

Summarizing the various models developed in sections (2.1)-(2.4), we obtain the following coupled fluid-structure interaction (FSI) problem:

$$
\begin{aligned}
v_{t} & =c^{2} u_{x x} \\
u_{t} & =v \\
u(x, 0) & =v(x, 0)=0 \\
m v_{t}(0, t) & =a P_{\mathrm{BLOOD}}(t)-k u(0, t)+\rho c^{2} a u_{x}(0, t) \\
v(L, t) & =-c u_{x}(L, t)
\end{aligned}
$$

where $P_{\text {BLOOD }}$ is given by $(20)$.

Next, we will present an analytical solution to eqs (23)-(27).

\section{Analytical Solution}

In order to simplify our work, we will rewrite eqs (23)-(27) in terms of only $u(x, t)$, which gives us:

$$
\begin{aligned}
u_{t t} & =c^{2} u_{x x} \\
u(x, 0) & =u_{t}(x, 0)=0 \\
m u_{t t}(0, t) & =a P_{\mathrm{BLOOD}}(t)-k u(0, t)+\rho c^{2} a u_{x}(0, t) \\
u_{t}(L, t) & =-c u_{x}(L, t)
\end{aligned}
$$

where $P_{\text {BLood }}$ is given by (20). Now, consider the Laplace Transform [6] of the dispacement $u(x, t)$ defined as:

$$
\mathscr{L}\{u(x, t)\}=U(x, s)=\int_{0}^{\infty} e^{-s t} u(x, t) d t .
$$

We will take the following approach to solve (28)-(31). By taking the Laplace Transform of (28)-(31), the PDE's are transformed into ordinary differential equations (ODE's) making it possible to solve for an exact solution for $U(x, s)$ at the 
point $x=0$. Then by taking the inverse Laplace Transform of $U(0, s)$ we find the exact solution for the movement of the outer wall $u(0, t)$.

The Laplace Transform of the wave equation (28) is:

$$
s^{2} U(x, s)=c^{2} U_{x x}(x, s) .
$$

This ODE has the solution:

$$
U(x, s)=c_{1} \cosh \left(\frac{s}{c} x\right)+c_{2} \sinh \left(\frac{s}{c} x\right)
$$

which is known up to the two constants, $c_{1}$ and $c_{2}$. To find them we first take the Laplace Transform of the boundary condition at the point $x=L$ (31) which gives

$$
s U(L, s)=-c U_{x}(L, s)
$$

Substituting (33) into (34) yields

$$
c_{2}=-c_{1}
$$

Next we take the Laplace transform of the boundary equation at the point $x=0$ (30) which gives:

$$
\begin{aligned}
m s^{2} U(0, s)= & \frac{a P_{m}}{s}-k U(0, s)+\rho c^{2} a U_{x}(0, s) \\
& +\sum_{n=1}^{N}\left(a A_{n}\left(\frac{s}{s^{2}+(n \omega)^{2}}\right)+a B_{n}\left(\frac{n \omega}{s^{2}+(n \omega)^{2}}\right)\right) .
\end{aligned}
$$

Substituting (33) into (35) and we find

$$
U(0, s)=\frac{\frac{a P_{m}}{s}+\sum_{n=1}^{N}\left(a A_{n}\left(\frac{s}{s^{2}+(n \omega)^{2}}\right)+a B_{n}\left(\frac{n \omega}{s^{2}+(n \omega)^{2}}\right)\right)}{m s^{2}+\rho c a s+k}
$$

Taking the inverse Laplace Transform [6] of (36) we find that:

$$
\begin{aligned}
u(0, t)= & A+B e^{r_{1} t}+C e^{r_{2} t} \\
& +\sum_{n=1}^{N}\left(D_{n} \cos (n \omega t)+\frac{E_{n}}{n \omega} \sin (n \omega t)+F_{n} e^{r_{1} t}+G_{n} e^{r_{2} t}\right)
\end{aligned}
$$


where:

$$
\begin{aligned}
r_{1,2} & =-\frac{\rho c a \pm \sqrt{(\rho c a)^{2}-4 m k}}{2 m} \\
A & =\frac{a P_{m}}{m r_{1} r_{2}} \\
B & =-\frac{a P_{m}}{r_{1}\left(r_{2}-r_{1}\right) m} \\
C & =\frac{a P_{m}}{r_{2}\left(r_{2}-r_{1}\right) m} \\
D_{n} & =-F_{n}-G_{n} \\
E_{n} & =-r_{1} F_{n}-r_{2} G_{n} \\
F_{n} & =\frac{a A_{n}-m G_{n}\left(r_{2}^{2}+n^{2} \omega^{2}\right)}{m\left(r_{1}^{2}+n^{2} \omega^{2}\right)} \\
G_{n} & =\frac{a\left(r_{2} A_{n}+n \omega B_{n}\right)}{m\left(r_{2}-r_{1}\right)\left(r_{2}^{2}+n^{2} \omega^{2}\right)}
\end{aligned}
$$

Equation (37) describes the solution for the displacement of the CSF at the point $x=0$. But as the point $x=0$ is where the outer wall meets the CSF, (37) is the equation that describes the movement of the outer wall for all time $t \geq 0$.

Looking at our solution for $u(0, t)(37)$ we can make some observations about the behavior of this equation. Because of our initial conditions, we know that $u(0,0)=$ 0 . Note that (37) is a sum of periodic terms, exponential terms and a constant. As both the periodic terms and the constant term are bounded, a finite sum of these terms is also bounded. Therefore to understand what this function looks like as $t$ increases towards infinity we need to understand the contribution of exponential terms.

Note that all of the exponential terms are of the form $y e^{r t}$ where $y$ is some constant and $r=r_{1}$ or $r_{2}$ where

$$
r_{1,2}=-\frac{\rho c a \pm \sqrt{(\rho c a)^{2}-4 m k}}{2 m} .
$$

Note also that this places a restriction on our values for $\rho, c, a, m$ and $k$ as in order for $r_{1,2}$ to be real, we need

$$
(\rho c a)^{2} \geq 4 m k
$$

To determine if $r_{1,2}$ is positive or negative, first we observe that, due to how the problem was defined, all the constants in $r_{1,2}(\rho, c, a, m, k)$ are all positive values. So:

$$
\begin{array}{r}
\frac{\rho c a+\sqrt{(\rho c a)^{2}-4 m k}}{2 m}>0 \\
r_{1}=-\frac{\rho c a+\sqrt{(\rho c a)^{2}-4 m k}}{2 m}<0
\end{array}
$$


For $r_{2}$ consider the fact that $\sqrt{(\rho c a)^{2}-4 m k}<\rho c a$. Thus:

$$
\begin{aligned}
\rho c a-\sqrt{(\rho c a)^{2}-4 m k} & >0 \\
\frac{\rho c a-\sqrt{(\rho c a)^{2}-4 m k}}{2 m} & >0 \\
r_{2}=-\frac{\rho c a-\sqrt{(\rho c a)^{2}-4 m k}}{2 m} & <0
\end{aligned}
$$

So all the exponential terms will go to zero as $t$ becomes very large. The value of the exponential terms at the point $t=0$ will depend on the constant $y$. Thus for large values of $t$ only the bounded periodic terms and the constant will affect the graph of $u(0, t)$. So we expect the function to start at $u(0,0)=0$ and become periodic over time.

\section{An Implicit Finite Difference Solution Methodedgy}

In this section we will develop a new implicit finite difference scheme to solve the coupled system eqs (23)-(27). To rewrite the derivatives of $u(x, t)$ and $v(x, t)$ we first consider the following general second order finite difference approximations for some function $f(y)$, where $0 \leq y \leq Y$ :

$$
\begin{array}{rrr}
f^{\prime}(y) & =\frac{f(y+\Delta y)-f(y-\Delta y)}{2 \Delta y}+O\left(\Delta y^{2}\right) & \Delta y \leq y \leq Y-\Delta y \\
f^{\prime \prime}(y)=\frac{f(y+\Delta y)-2 f(y)+f(y-\Delta y)}{\Delta y^{2}}+O\left(\Delta y^{2}\right) & \Delta y \leq y \leq Y-\Delta y \\
f^{\prime}(0)=\frac{-3 f(0)+4 f(\Delta y)-f(2 \Delta y)}{2 \Delta y}+O\left(\Delta y^{2}\right) & (y=0) \\
f^{\prime}(Y)=\frac{f(Y-2 \Delta y)-4 f(Y-\Delta y)+3 f(Y)}{2 \Delta y}+O\left(\Delta y^{2}\right) & (y=Y)
\end{array}
$$

Figure 3 illustrates the computation domain for $u(x, t)$, where $0 \leq x \leq L$ and

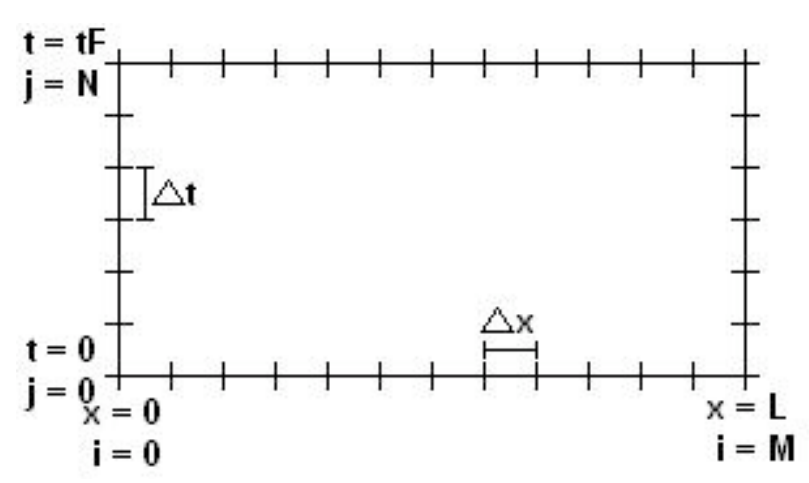

Figure 3. Computational domain

$0 \leq t \leq t F$, as a grid with the $x$ domain partitioned into $M$ subintervals, with spatial step size $\Delta x=\frac{L}{M}$, and the time partitioned into $N$ subintervals, with temporal step size $\Delta t=\frac{t F}{N}$. For simplicity of notation we will use $U_{j}^{i}=u((\Delta x) i,(\Delta t) j)$ and 
FSI MODELS FOR BIOLOGICAL SYSTEMS

$V_{j}^{i}=v((\Delta x) i,(\Delta t) j)$. Thus, eqs (23)-(24) are rewritten implicitly for $j \geq 1$ as:

$$
\frac{V_{i}^{j+1}-V_{i}^{j-1}}{2 \Delta t}=\frac{c^{2}\left(U_{i+1}^{j+1}-2 U_{i}^{j+1}+U_{i-1}^{j+1}\right)}{\Delta x^{2}}+O\left(\Delta x^{2}, \Delta t\right)
$$

for $1 \leq i \leq M-1$, and:

$$
\frac{U_{i}^{j+1}-U_{i}^{j-1}}{2 \Delta t}=V_{i}^{j+1}+O(\Delta t)
$$

for $0 \leq i \leq M$. Note that because we are using an implicit scheme, the right hand sides of both (38) and (39), while being second order in space, are first order in time. One can verify this using Taylor Series expansion in both space and time. The boundary conditions eqs (26) - (27) are rewritten for $j \geq 1$ as:

$$
\begin{aligned}
\frac{m\left(V_{0}^{j+1}-V_{0}^{j-1}\right)}{2 \Delta t}= & a P_{\mathrm{BLOOD}}(\Delta t(j+1))-k U_{0}^{j+1} \\
& +\frac{\rho c^{2} a\left(-3 U_{0}^{j+1}+4 U_{1}^{j+1}-U_{2}^{j+1}\right)}{2 \Delta x}+O\left(\Delta x^{2}, \Delta t\right)
\end{aligned}
$$

where $P_{\text {BLOOD }}$ is given by $(20)$, and:

$$
V_{M}^{j+1}=\frac{-c\left(U_{M-2}^{j+1}-4 U_{M-1}^{j+1}+3 U_{M}^{j+1}\right)}{2 \Delta x}+O\left(\Delta x^{2}\right)
$$

By gathering all $j+1$ terms on one side and dropping higher order terms, (38)-(41) becomes:

PDE for $j \geq 1$ :

$$
\begin{gathered}
\left(\frac{2 c^{2}}{\Delta x^{2}}\right) U_{i}^{j+1}-\left(\frac{c^{2}}{\Delta x^{2}}\right)\left(U_{i-1}^{j+1}+U_{i+1}^{j+1}\right)+\left(\frac{1}{2 \Delta t}\right) V_{i}^{j+1}=\left(\frac{1}{2 \Delta t}\right) V_{i}^{j-1} \\
U_{i}^{j+1}-2 \Delta t V_{i}^{j+1}=U_{i}^{j-1}
\end{gathered}
$$

Boundary Conditions for $j \geq 1$ :

$$
\begin{aligned}
\left(k+\frac{3 \rho c^{2} a}{2 \Delta x}\right) U_{0}^{j+1}-\left(\frac{2 \rho c^{2} a}{\Delta x}\right) U_{1}^{j+1}+\left(\frac{\rho c^{2} a}{2 \Delta x}\right) U_{2}^{j+1} & +\left(\frac{m}{2 \Delta t}\right) V_{0}^{j+1} \\
& =\left(\frac{m}{2 \Delta t}\right) V_{0}^{j-1}+a P_{\text {BLOOD }}(\Delta t(j+1))
\end{aligned}
$$

$$
c U_{M-2}^{j+1}-4 c U_{M-1}^{j+1}+3 c U_{M}^{j+1}+2 \Delta x V_{M}^{j+1}=0
$$

In matrix form this may be written as:

$$
A\left[\begin{array}{c}
U_{0} \\
U_{1} \\
\vdots \\
U_{M-1} \\
U_{M} \\
V_{0} \\
V_{1} \\
\vdots \\
V_{M-1} \\
V_{M}
\end{array}\right]^{j+1)}=\left[\begin{array}{c}
\frac{m}{2 \Delta t} V_{0} \\
\frac{1}{2 \Delta t} V_{1} \\
\vdots \\
\frac{1}{2 \Delta t} V_{M-1} \\
0 \\
U_{0} \\
U_{1} \\
\vdots \\
U_{M-1} \\
U_{M}
\end{array}\right]^{j-1)}+\left[\begin{array}{c}
a P_{\mathrm{BLOOD}}(\Delta t(j+1)) \\
0 \\
0 \\
0 \\
0 \\
\vdots \\
0 \\
0 \\
0 \\
0
\end{array}\right]
$$


where:

$$
\begin{aligned}
& A=\left[\begin{array}{ll}
B & C \\
D & E
\end{array}\right] \\
& B=\left[\begin{array}{cccccc}
k+\frac{3 \rho c^{2} a}{2 \Delta x} & \frac{-2 \rho c^{2} a}{\Delta x} & \frac{\rho c^{2} a}{2 \Delta x} & 0 & \cdots & 0 \\
-\frac{c^{2}}{\Delta x^{2}} & 2 \frac{c^{2}}{\Delta x^{2}} & -\frac{c^{2}}{\Delta x^{2}} & 0 & \cdots & 0 \\
0 & -\frac{c^{2}}{\Delta x^{2}} & 2 \frac{c^{2}}{\Delta x^{2}} & -\frac{c^{2}}{\Delta x^{2}} & \cdots & 0 \\
\vdots & & \ddots & \ddots & \ddots & \vdots \\
0 & \ldots & 0 & -\frac{c^{2}}{\Delta x^{2}} & 2 \frac{c^{2}}{\Delta x^{2}} & -\frac{c^{2}}{\Delta x^{2}} \\
0 & \ldots & 0 & c & -4 c & 3 c
\end{array}\right] \\
& C=\left[\begin{array}{ccccc}
\frac{m}{2 \Delta t} & 0 & 0 & \cdots & 0 \\
0 & \frac{1}{2 \Delta t} & 0 & \cdots & 0 \\
\vdots & & \ddots & & \vdots \\
0 & \cdots & 0 & \frac{1}{2 \Delta t} & 0 \\
0 & \cdots & 0 & 0 & 2 \Delta x
\end{array}\right] \\
& D=\left[\begin{array}{cccc}
1 & 0 & \cdots & 0 \\
0 & 1 & \cdots & 0 \\
\vdots & & \ddots & \vdots \\
0 & \cdots & 0 & 1
\end{array}\right] \\
& E=\left[\begin{array}{cccc}
-2 \Delta t & 0 & \cdots & 0 \\
0 & -2 \Delta t & \cdots & 0 \\
\vdots & & \ddots & \vdots \\
0 & \cdots & 0 & -2 \Delta t
\end{array}\right]
\end{aligned}
$$

In order to solve this system (46), we first need solutions at the first two time levels, $j=0$ and $j=1$. For $j=1$, we use the first order finite difference approximation

$$
f^{\prime}(y)=\frac{f(y+\Delta y)-f(y)}{\Delta y}+O(\Delta y) .
$$

Using this approximation, for $j=1(23)-(24)$ are rewritten implicitly as:

$$
\frac{V_{i}^{1}-V_{i}^{0}}{\Delta t}=\frac{c^{2}\left(U_{i+1}^{1}-2 U_{i}^{1}+U_{i-1}^{1}\right)}{\Delta x^{2}}+O\left(\Delta x^{2}, \Delta t\right)
$$

for $1 \leq i \leq M-1$, and:

$$
\frac{U_{i}^{1}-U_{i}^{0}}{\Delta t}=V_{i}^{1}+O(\Delta t)
$$

for $0 \leq i \leq M$. The boundary conditions (26) - (27) are rewritten implicitly as:

(49) $\frac{m\left(V_{0}^{1}-V_{0}^{0}\right)}{\Delta t}=a P_{\mathrm{BLOOD}}(\Delta t)-k U_{0}^{1}+\frac{\rho c^{2} a\left(-3 U_{0}^{1}+4 U_{1}^{1}-U_{2}^{1}\right)}{2 \Delta x}+O\left(\Delta x^{2}, \Delta t\right)$

where $P_{\text {BLOOD }}$ is given by $(20)$, and:

$$
V_{M}^{1}=\frac{-c\left(U_{M-2}^{1}-4 U_{M-1}^{1}+3 U_{M}^{1}\right)}{2 \Delta x}+O\left(\Delta x^{2}\right)
$$

From the initial conditions (25) we know that at $j=0, U_{i}^{0}=V_{i}^{0}=0$ for $0 \leq i \leq M$. By substituting this into (47)-(50), dropping higher order terms, and rewriting in 
matrix form we get:

$$
F\left[\begin{array}{c}
U_{0} \\
U_{1} \\
\vdots \\
U_{M-1} \\
U_{M} \\
V_{0} \\
V_{1} \\
\vdots \\
V_{M-1} \\
V_{M}
\end{array}\right]^{(1)}=\left[\begin{array}{c}
\left.a P_{\mathrm{BLOOD}}(\Delta t)\right) \\
0 \\
0 \\
0 \\
0 \\
\vdots \\
0 \\
0 \\
0 \\
0
\end{array}\right]
$$

where:

$$
\begin{aligned}
F & =\left[\begin{array}{cc}
B & G \\
D & H
\end{array}\right] \\
G & =\left[\begin{array}{ccccc}
\frac{m}{\Delta t} & 0 & 0 & \cdots & 0 \\
0 & \frac{1}{\Delta t} & 0 & \cdots & 0 \\
\vdots & & \ddots & & \vdots \\
0 & \cdots & 0 & \frac{1}{\Delta t} & 0 \\
0 & \cdots & 0 & 0 & 2 \Delta x
\end{array}\right] \\
H & =\left[\begin{array}{cccc}
-\Delta t & 0 & \cdots & 0 \\
0 & -\Delta t & \cdots & 0 \\
\vdots & & \ddots & \vdots \\
0 & \cdots & 0 & -\Delta t
\end{array}\right]
\end{aligned}
$$

Thus by solving (51) we get the solution for $j=1$. Then for $j \geq 2$ we solved (46).

\section{Computational Experiments}

In this section, we perform the following computational studies. First we validate the numerical solution obtained implicitly by solving eq (46) against the analytical solution for $u(0, t)$ eq $(37)$. Once our model is validated we study the influence of various parameters on the displacement of the wall $u(0, t)$.

We consider the following realistic values for our experiments. For the CSF, we used $\rho=1000 \mathrm{~kg} / \mathrm{m}^{3}[2]$ and $c=1500 \mathrm{~m} / \mathrm{s}$. For the blood pressure model, we used $P_{m}=65.7 \mathrm{mmHg}, \omega=1 \mathrm{rad} / \mathrm{s}$, and for the harmonics we used $A_{1}=-7.13, B_{1}=$ 4.64, $A_{2}=-3.08, B_{2}=-1.18, A_{3}=-0.130, B_{3}=-0.564, A_{4}=-0.205, B_{4}=$ $-0.346, A_{5}=0.0662, B_{5}=-0.120$, all in $\mathrm{mmHg}$ [16]. Finally for the wall we used $a=.01 \mathrm{~m}^{2}, k=8000 \mathrm{~N} / \mathrm{m}$ and $m=.001 \mathrm{~kg}$. Note that these values meet our restriction from the analytical solution that $(\rho c a)^{2} \geq 4 m k$ as:

$$
\begin{aligned}
(\rho c a)^{2} & =\left[1000\left(\mathrm{~kg} / \mathrm{m}^{3}\right) * 1500(\mathrm{~m} / \mathrm{s}) * .01\left(\mathrm{~m}^{2}\right)\right]^{2} \\
& =2.25 * 10^{8}\left(\mathrm{~kg}^{2} / \mathrm{s}^{2}\right) \\
& \geq 4 \mathrm{mk} \\
& =4 * .001(\mathrm{~kg}) * 8000(\mathrm{~N}) \\
& =32\left(\mathrm{~kg}^{2} / \mathrm{s}^{2}\right) .
\end{aligned}
$$




\section{S. MINERVA VENUTI}

In addition, the eigenvalues of matrix $A$ from eq (46) were computed which resulted in all nonzero values.

5.1. Comparison of Analytical vs Numerical. In Figure 4 presents the analytical and numerical solutions for the displacement of the outer wall $u(0, t)$. Due to our intial conditions the displacement starts at zero and stabilizes after a few seconds. Note that, as we predicted from our analytical solution, the exponential terms seem to affect the graph in the first few seconds, as the graph quickly increases from zero, but then the graph stabilizes into a bounded periodic motion. It is clear from Figure 4 that the implicit finite difference scheme developed for the coupled system matches well with the analytical solution.

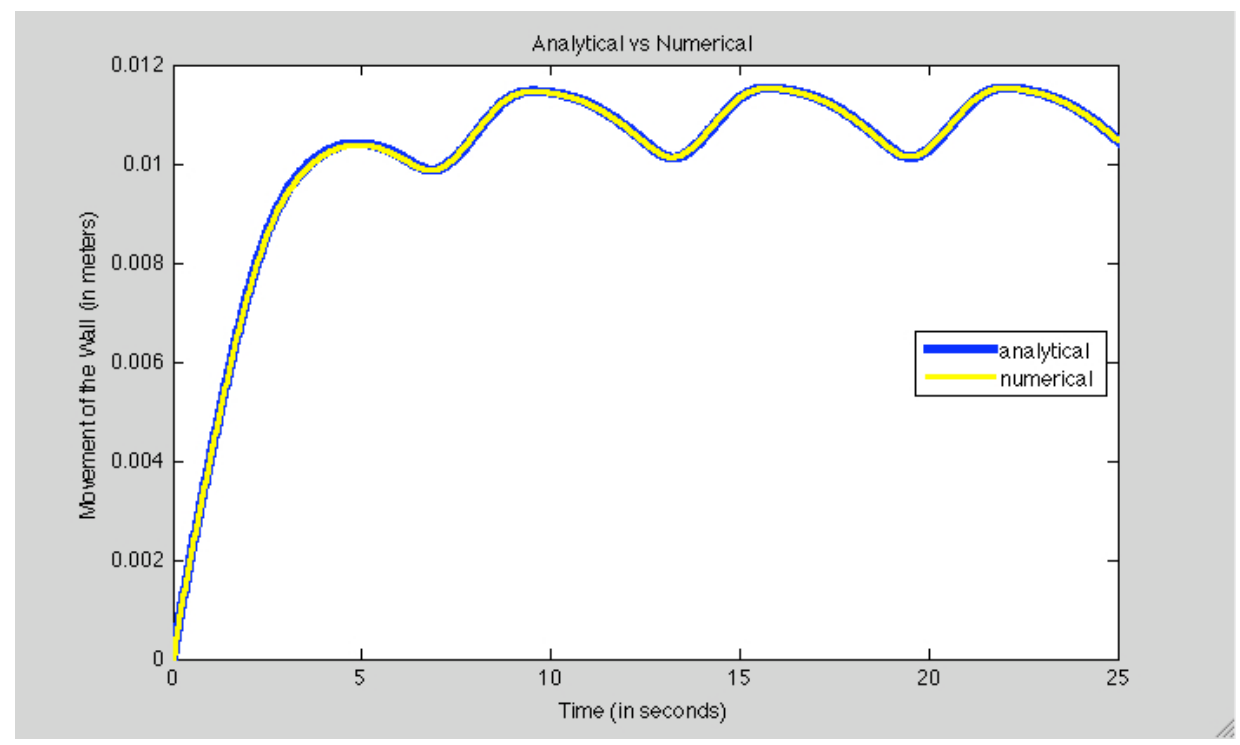

Figure 4. Analytical vs Numerical

In order to determine the rate of convergence of the numerical scheme, we first assume that there is a power relationship between $\Delta t$ and the relative $L_{2}$ norm error, $e$. Thus $e \approx c(\Delta t)^{\alpha}$ where $c$ is a constant. To find $\alpha$ :

$$
\begin{aligned}
e_{1} & \approx c\left(\Delta t_{1}\right)^{\alpha} \\
e_{2} & \approx c\left(\Delta t_{2}\right)^{\alpha} \\
\frac{e_{1}}{e_{2}} & \approx\left(\frac{\Delta t_{1}}{\Delta t_{2}}\right)^{\alpha} \\
\alpha & \approx \frac{\ln \frac{e_{1}}{e_{2}}}{\ln \frac{\Delta t_{1}}{\Delta t_{2}}}
\end{aligned}
$$

See Table 1 for $\alpha$ values calculated as the step size $\Delta t$ is reduced. The value of $\alpha$ approaches 1 , indicating that it is first order in time. 
FSI MODELS FOR BIOLOGICAL SYSTEMS

\begin{tabular}{|c|c|c|}
\hline$\Delta t$ & Relative $L_{2}$ Norm & $\alpha$ \\
\hline 12.5000 & 0.7165 & - \\
\hline 6.2500 & 0.5086 & 0.4945 \\
\hline 3.1250 & 0.3420 & 0.5725 \\
\hline 1.5625 & 0.1741 & 0.9741 \\
\hline 0.7812 & 0.0739 & 1.2366 \\
\hline 0.3906 & 0.0337 & 1.1341 \\
\hline 0.1953 & 0.0160 & 1.0773 \\
\hline 0.0977 & 0.0077 & 1.0437 \\
\hline 0.0488 & 0.0038 & 1.0233 \\
\hline 0.0244 & 0.0019 & 1.0117 \\
\hline
\end{tabular}

TABLE 1. Error for decreasing $\Delta t$.

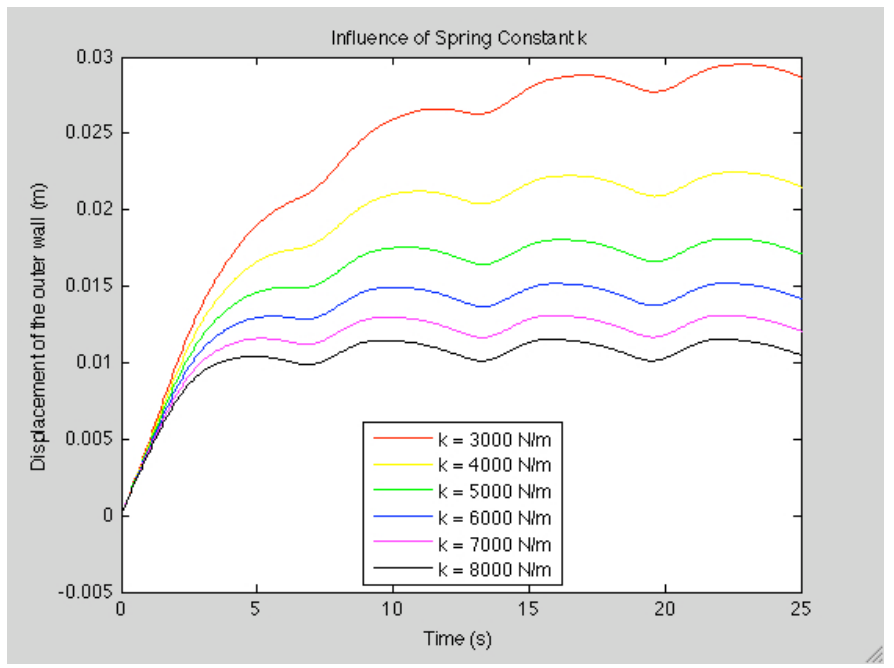

FiguRE 5. Influence of wall stiffness
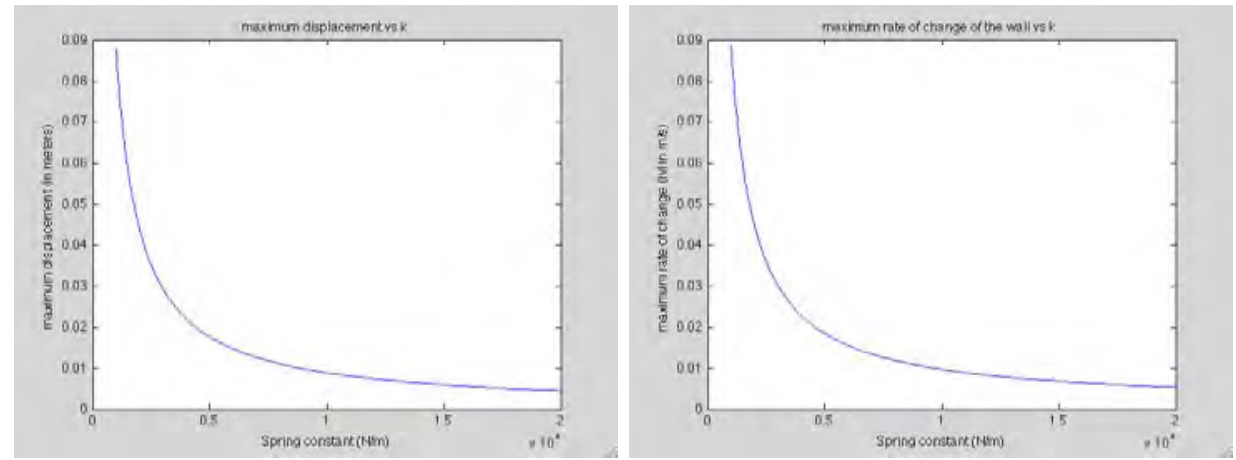

FiguRe 6. Influence of wall stiffness on maximum displacement and maximum velocity.

5.2. Influence of the Stiffness of the Wall. The spring constant physically models the stiffness of the arterial wall. Therefore, as the spring constant is decreased, the wall becomes more flexible, which causes the wall to move father out and to take longer to stabilize. Both Figures 5 and 6 illustrates this. In Figure 


\section{S. MINERVA VENUTI}

5 we plot the solution for decreasing values of the spring constant from $k=8000$ $\mathrm{N} / \mathrm{m}$ to $k=3000 \mathrm{~N} / \mathrm{m}$. We can see that as $k$ decreases the displacement is greater and it takes longer for the wall to settle into a steady periodic motion. However, note that the value of the spring constant has no affect on the amplitude of the periodic movement of the wall. In Figure 6 we plot the maximum displacement and maximum velocity reached by the wall for varying values of $k$. Again we notice that the maximum displacement decreases as $k$ increases, as does the maximum velocity of the wall.

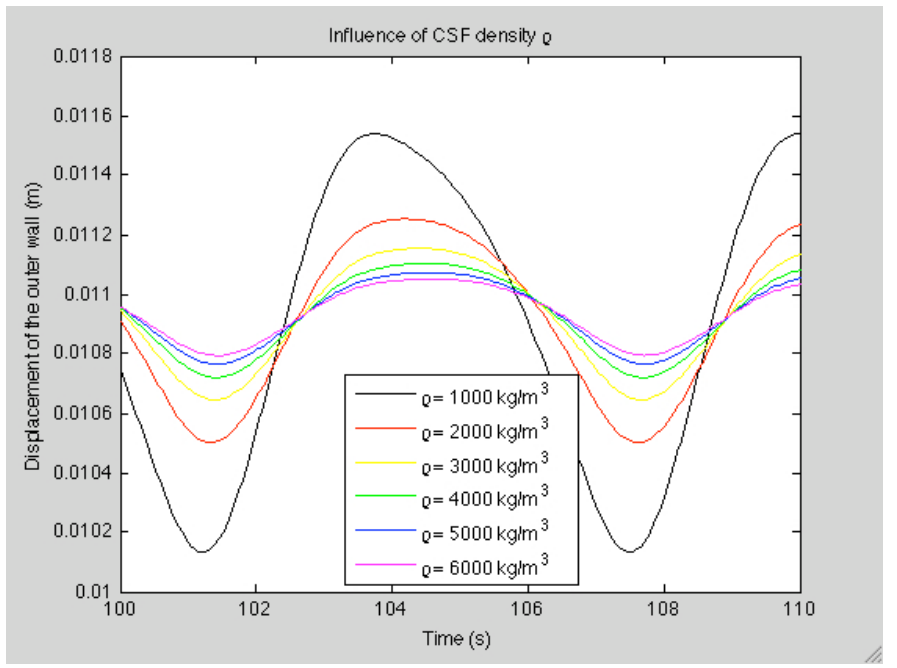

FiguRE 7. Influence of density of CSF.
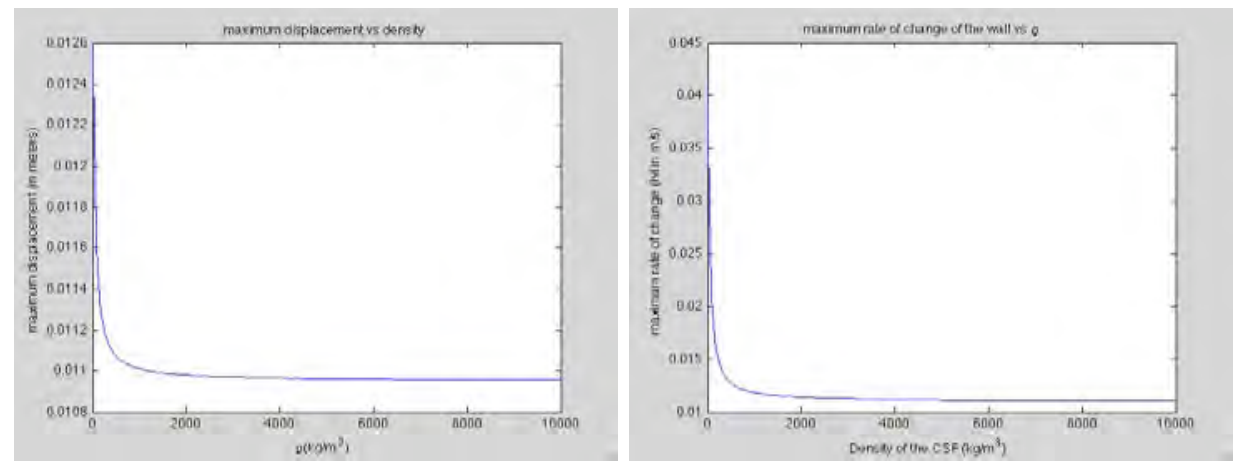

FIGURE 8. Influence of density of CSF on maximum displacement and maximum velocity.

5.3. Influence of the Density of the CSF. As the CSF becomes more dense, it resists the movement of the wall, so the amplitude of the periodic movement of the wall is expected to become much less. Both Figures 7 and 8 show this. Figure 7 illustrates the motion of the wall for increasing values of the density from $\rho=1000$ $\mathrm{kg} / \mathrm{m}^{3}$ to $\rho=6000 \mathrm{~kg} / \mathrm{m}^{3}$ of the CSF. Note that this graph is at a later time period than Figure 5; it is after the movement of the wall has stabilized. Also note that the maximum and minimum points of the wall's movement shift to a later time as the CSF becomes more dense, showing that it takes longer for the wall to push the CSF aside. In Figure 7 we are graphing the maximum displacement 
and maximum velocity as $\rho$ is increased, and on the graphs we see that they both decrease quickly as $\rho$ is increased from close to zero to about $2000 \mathrm{~kg} / \mathrm{m}^{3}$, and then remain at constant values.

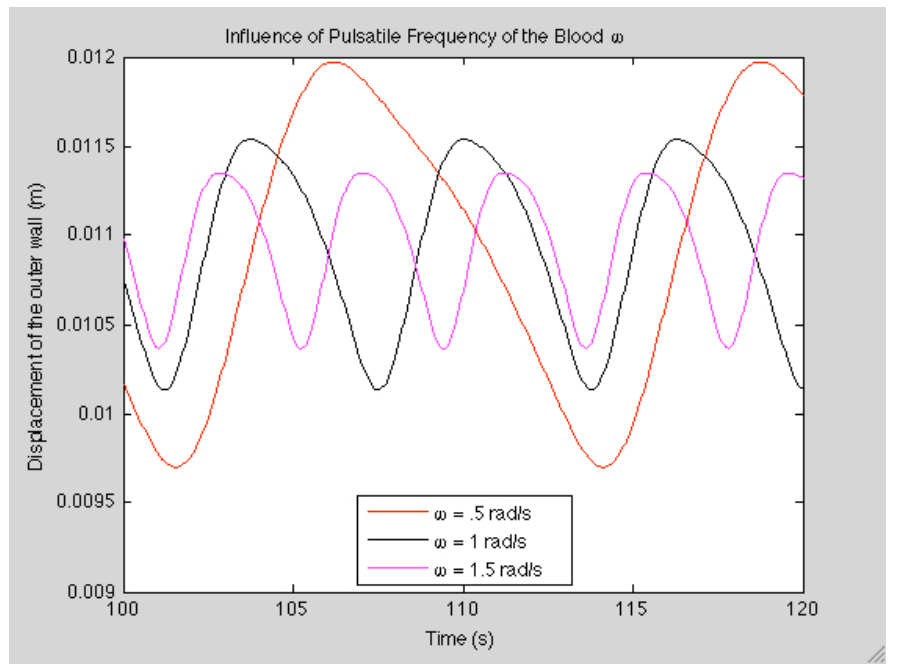

FIGURE 9. Influence of pulsatile frequency of the blood
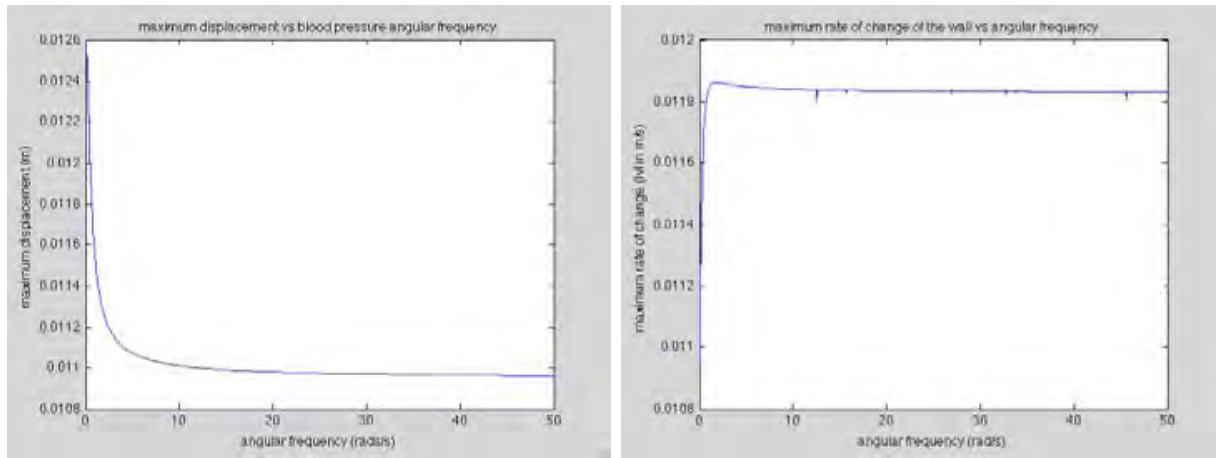

FIGURE 10. Influence of pulsatile frequency of the blood on maximum displacement and maximum velocity.

5.4. Influence of Pulsatile Frequency of the Blood. As the frequency is increased, the period of the periodic movement of the wall is expected to decrease, as is the amplitude. To verify this, the motion of the wall was investigated for different values of the pulsatile frequency. In Figure 9 the pulsatile frequency of the blood pressure $(\omega)$ is varied from $\omega=0.5$ to $\omega=1.5$. By looking at eq (37) we know that the frequency of the periodic motion depends only on $\omega$ so it makes sense that the period decreases as frequency increases. The fact that increasing the frequency results in the amplitude of the wall decreases means that the outer wall has less time to react to the pressure from the blood pushing before it switches directions, thus the lower amplitude. Also in Figure 10 we have plotted the maximum displacement and maximum velocity as a function of $\omega$. As with the density of the CSF, when $\omega$ increases, the maximum displacement decreases. However the velocity increases to a maximum of $0.0119 \mathrm{~m} / \mathrm{s}$, which corresponds to $\omega=1.95 \mathrm{rads} / \mathrm{s}$, then decreases slightly to a constant value. 


\section{S. MINERVA VENUTI}

\section{Conclusions And Future Work}

This work has been about creating a computational model of a complex biological system that incorporates all three major aspects of an aneurysm: the CSF, the arterial wall and the blood pressure. To provide a better insight into the complex model a simple one dimensional model was formulated and an analytical solution was derived. This was done primarily by making a number of assumptions about the CSF to simplify the associated PDE (1) to the wave equation (17)-(18). In addition we developed a numerical scheme to solve the same simplified model. Now that we have both this analytical solution and a numerical methodology that matches the analytical solution, our next goal is to relax some of the assumptions made and solve the associated coupled model using the numerical methodology developed in this paper. This will be the focus of a forthcoming paper.

\section{ACKNOWLEDGEMENTS}

This work is supported in part by the National Science Foundation, the George Mason Undergraduate Apprenticeship Program and the National Institutes of Heath. The author wishes to acknowledge the following individuals Dr. Javed Siddque, Mr. Kevin Kelbaugh, Ms Courtney Chancellor and Ms Kris Kappemeyer for useful discussions on this subject. The author also wishes to thank the judges of the Second Annual SIAM Undergraduate Poster Session held at Denver, Colorado on July 7 th, 2009 where this paper was presented.

\section{REFERENCES}

[1] E. Aulisa, S. Manservisi and P. Seshaiyer. A computational multilevel approach for solving 2D Navier-Stokes equations over non-matching grids. Computer Methods in Applied Mechanics and Engineering, vol 195, pp 4604-4616, 2006.

[2] H.L. Brydon, R. Hayward, W. Harkness, and R. Bayston. Physical properties of cerebrospinal fluid of relevance to shunt formation. The effect of protein upon CSF. British Journal of Neurosurgery 9, 639-644, 1995.

[3] P.B. Crompton. Mechanism of growth and rupture in cerebral berry aneurysms. Br. Med J. $11138-1142,1966$.

[4] G.G. Ferguson. Direct measurement of mean and pulsatile blood pressure at operation in human intracranial saccular aneurysms. Journal of Neurosurgery 36, 560-563, 1972.

[5] L. Ferguson, E. Aulisa, P. Seshaiyer. Computational modeling of highly flexible membrane wings in micro air vehicles. Proceddings of the 47th AIAA/ASME/ASCE/AHS/ASC Structures, Structural Dynamics, and Meterials Conference Newport, RI (2006).

[6] R. Haberman. Elementary Applied Partial Differential Equations: With Fourier Series and Boundary Value Problems, Third Edition. Prentice Hall, Upper Saddle River, NJ, 1998.

7] N. Hashimoto and H. Handa. The size of cerebral aneurysms in relation to repeated rupture. Sury. Neurol,19, 107-111, 1983.

[8] J.D. Humphrey. Arterial wall mechanics: review and directions. Critical Reviews in Biomedical Engineering 23, 1-162, 1995.

[9] J.J. Jain. Mechanism of rupture in intracranial saccular aneurysms. Surgery 54, 347-350, 1963.

[10] N.F. Kassell and J.C. Torner. Size of intracranial aneurysms. Neurosurgery, 12 291-297, 1983.

[11] W.R. Milnor. Hemodynamics. Williams \& Wilkens, Baltimore, 1982.

[12] L.N. Sekhar and R.C. Heros. Origin, growth and rupture of saccular aneurysms; a review. Neurosurgery 8, 248-260, 1981.

[13] L.N. Sekhar, R.J. Sclabassi, M. Sun, H.B. Blue, J.F. Wasserman. Intra-aneurysmal pressure measurements in experimental saccular aneurysms in dogs. Stroke 19, 352-356, 1988.

[14] P. Seshaiyer and J.D. Humphrey. On the protective role of contact constraints in saccular aneurysms. Journal of Biomechanics, 34: 607-612, 2001. 
FSI MODELS FOR BIOLOGICAL SYSTEMS

[15] P. Seshaiyer and J.D. Humphrey. A sub-domain inverse finite element characterization of hyperelastic membranes including soft tissues. ASME Journal of Biomechanical Engineering, 125(3): 363-371, 2003.

[16] A.D. Shah and J.D. Humphrey. Finite strain elastodynamics of intracranial saccular aneurysms. Journal of Biomechanics 32 593-599, 1999.

[17] T.E. Simkins and W.E. Stehbens. Vibrational behavior of arterial aneurysms. Letters in Applied and Engineering Sciences 1, 85-100, 1973.

[18] E.W. Swim and P. Seshaiyer. A nonconforming finite element method for fluid-structure interaction problems. Computer Methods in Applied Mechanics and Engineering vol. 195(1718), pp. 2088-2099, 2006. 